\title{
Factores Relacionados con la Utilización de Servicios de Salud en Costa Rica
}

\section{Factors related to using health services in Costa Rica}

\author{
Amada A. Llanos ${ }^{1,3}$, Melvin Morera-Salas ${ }^{1}$, Patricia Barber-Pérez ${ }^{2}$, \\ Karla Hernández ${ }^{3}$, Yanira Xirinach-Salazar ${ }^{3}$ y Juan R. Varga ${ }^{3}$ \\ 1 Caja Costarricense de Seguro Social. amadaaparicio.llanos@gmail.com \\ 2 Universidad Las Palmas de Gran Canarias, España \\ 3 Universidad de Costa Rica-Centro Centroamericano de Población \\ Recibido 10 Noviembre 2008/Enviado para Modificación 14 Febrero 2009/Aceptado 10 Mayo 2009
}

\section{RESUMEN}

Objetivo Determinar el comportamiento de los costarricenses en la utilización de los servicios de salud.

Métodos Los datos se obtuvieron de la primera Encuesta Nacional de Salud de Costa Rica. En el análisis de los datos se utilizaron medidas de tendencia central, dispersión, proporciones, frecuencias, prueba de Ji-cuadrado y de Kruskal-Walis.

Resultados Tanto en los servicios de consulta ambulatoria y urgencias los factores que predisponen su utilización son, la región de residencia y el nivel de ingreso, mientras que en los servicios de hospitalización es el nivel educativo. El aseguramiento y la región de residencia facilitan el uso de la consulta ambulatoria y la hospitalización. La salud autopercibida, permanecer en cama al menos medio día y padecer alguna enfermedad crónica son variables de necesidad asociadas al uso de consulta ambulatoria; la enfermedad crónica se asocia al uso del servicio de hospitalización y el permanecer en cama al uso del servicio de urgencia.

Discusión La mayoría de variables que postula el modelo de Andersen y Newman sobre la utilización de servicios de salud, se cumplen para Costa Rica. Sin embargo, la significancia de los factores varía entre los servicios de consulta ambulatoria, hospitalización y urgencias.

Palabras Clave: Servicios de salud, estado de salud, percepción (fuente: DeCS, BIREME).

\section{ABSTRACT}

Objective Determining Costa Ricans' behaviour patterns when using health services. Methods The Costa Rican Health Survey was used. Central tendency, dispersion, percentages, frequencies, Ji-Square and Kruskal-Walis test measurements were analysed.

Results Area of residence and income level were the predisposing factors in both out-patient and emergency services whereas educational level was so in terms of hospitalisation service. Health insurance status and the area of residence were the enabling factors associated with using out-patient and hospitalisation services. The 
need factors associated with outpatient services were the individuals' perceived state of health, having remained in bed at least until noon and suffering some chronic disease; chronic disease was associated with hospitalisation and remaining in bed when using the emergency service.

Discussion Most variables postulated by Andersen and Newman's model as being determinants for using health services were present in Costa Rica. Nevertheless, the significance of its factors varied between outpatient, hospitalisation and emergency services.

Key Words: Health service, health status, perception (source: MeSH, NLM).

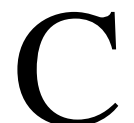

osta Rica tenía una población de 4.4 millones de habitantes en 2007, una tasa de natalidad de 16 por mil y de mortalidad general de 3,8 por mil habitantes y una esperanza de vida general de 79 años. Su sistema de salud es financiado en un $75 \%$ por el sector público, el cual está formado por un conjunto de instituciones entre las que se encuentra la Caja Costarricense de Seguro Social (CCSS) que acumula el $80 \%$ del presupuesto de salud público y cubre un $86 \%$ de la población del país (1). Por su parte en el sector privado no existen seguros de salud formales, con lo cual el pago de servicios privados de salud se convierte en pago de bolsillo.

El conocer los determinantes que afectan la utilización de servicios de salud, es un elemento clave en el diseño de políticas de salud, ya que cambios en estos pueden afectar las estimaciones presupuestarias del seguro público, la planificación de la cartera de servicios, así como consideraciones de equidad y sostenibilidad del seguro de salud.

En el país se han realizado pocos estudios sobre utilización de servicios de salud los cuales abordan principalmente la proporción de utilización en el sector público y privado, pero no se han abordado los factores asociados a dicha utilización $(2,3)$.

Dada esta situación, el presente estudio trata de avanzar en este análisis, abordando los determinantes bajo un enfoque que permita dar explicación a patrones de utilización de servicios de salud, como es el caso del modelo de Andersen y Newman $(4,5)$.

Este modelo consiste en relacionar tres tipos de factores para dar explicación a los patrones de utilización de los servicios de salud basados en la predisposición para utilizar los servicios (factores predisponentes), capacidad para obtenerlos (factores facilitadores) y nivel de enfermedad (factores de necesidad). 
Los factores predisponentes incluyen características sociodemográficas entre las que podemos citar la edad, el sexo, la educación, ocupación, clase social y actividad laboral (6-8). En la edad los grupos etáreos extremos usan más servicios, lo que es congruente con el incremento en morbilidad y mortalidad de este grupo de población (9). El sexo femenino es el que mayormente utiliza los servicios dado los episodios no mórbidos de salud en edad reproductiva (10). El mayor nivel de escolaridad se asocia a mayor conocimiento de la disponibilidad de servicios de atención y beneficios en el estado de salud (11). La ocupación se asocia a mayor utilización según los perfiles de morbilidad, y accidentes laborales (12). La utilización según clase social se asocia al nivel de ingreso (13) y los programas preferenciales dirigidos a poblaciones socioeconómicamente deprimidas (14).

Los factores facilitadores se refieren a condiciones que garantizan la disponibilidad y acceso a servicios de salud. Entre los que se incluyen características individuales de nivel de ingreso o tipo de seguro, características poblacionales de disponibilidad y accesibilidad de servicios (15), tipo de seguro médico en individuos con seguro, financieramente más capaces de adquirir servicios sin erogar directamente ninguna cantidad (16) y nivel de ingreso asociado a la superación o no de los obstáculos financieros (17).

Los factores de necesidad representan una de las causas más inmediatas del uso de los servicios, varía en función de los factores predisponentes y facilitadores. Habitualmente se evalúa según el estado de salud percibido u otras variables relacionadas con la salud, como son los días en la cama o la restricción de la actividad aguda o crónica (18).

Según el servicio que se utilice, se ha descrito en muchos países que las consultas médicas y las hospitalizaciones son más utilizados por personas de clase social baja (7). Y que la existencia de antecedentes patológicos, el número de patologías asociadas, la discapacidad física y una baja percepción del estado de salud se asocian con mayor frecuencia a usos de los servicios de urgencias (8).

Al relacionar utilización y necesidad se ha encontrado que para un mismo nivel de necesidad, las personas de clase social más desaventajadas tienen realmente un menor acceso a los servicios de salud (7).

Este estudio tiene como objetivo identificar el comportamiento de los costarricenses en la utilización de los servicios de salud y su relación con variables de predisposición a utilizarlos, las condiciones que facilitan su uso y la necesidad de utilizarlos. 


\section{MATERIALES Y MÉTODOS}

En las últimas décadas se ha observado una marcada tendencia al uso de las encuestas de la salud auto reportada, para analizar los patrones de demanda y utilización de servicios de salud (19-21).

Para realizar el presente trabajo se utilizaron los datos de la primera Encuesta Nacional de Salud para Costa Rica 2006 (22), estudio transversal llevado a cabo en una muestra de 7523 personas representativa de la población no institucionalizada residente en Costa Rica.

El análisis se realizó con el número de sujetos que respondió a cada variable analizada, es decir se eliminaron las observaciones con datos perdidos en las variables relevantes.

Una de las ventajas de esta encuesta es que proporciona información a nivel nacional y por región, sobre la valoración del estado de salud de los individuos, permite conocer el grado de utilización de los servicios de salud, sus características, el uso de determinadas prácticas preventivas, la frecuencia y distribución de hábitos de vida que suponen riesgo para la salud y permite relacionar esta información con las características sociodemográficas de la población.

La unidad de análisis son las personas de todas las edades. El evento analizado es haber consultado con el médico en el último mes, haber estado hospitalizado al menos un día en el último año y haber utilizado algún servicio de urgencias en el último año.

Los servicios de atención en salud conocidos como consultas médicas, hospitalización y urgencias, se asociaron con las tres categorías de variables que propone el modelo de Andersen y Newman (4), para explicar las diferencias en utilización.

Se realizó un análisis descriptivo -medidas de tendencia central, dispersión, proporciones y frecuencias-, test de Ji-Cuadrado para variables cualitativas dicotómicas y análisis de homogeneidad; test de Kruskal-Walis para las variables cuantitativas no normales, y ANOVA para aquellas cuya distribución puede aceptarse normal. Los resultados se consideraron significativos cuando el estadístico resultara menor que $0.05(\mathrm{p}<0,5)$. Los datos se procesaron en SPSS 10.0. 


\section{RESULTADOS}

La población analizada se distribuyó de la siguiente manera, el $52 \%$ de los encuestados eran hombres, el $65 \%$ residían en zona urbana, el $58 \%$ de la muestra tenía entre 20 y 64 años, el $38 \%$ vivían pareja, el $65 \%$ se ubicaban en la región central de país, 70 \% habían cursado hasta secundaria incompleta y un $6 \%$ tenían hasta universidad completa $22 \%$ en el quintil I y $17 \%$ en el quintil V y un $87 \%$ de los encuestados cuentan con seguro.

Tabla 1. Uso del servicio de consulta ambulatoria y significancia observada, según variables predisponentes

\begin{tabular}{|c|c|c|c|c|c|}
\hline Variable & Categoría & Número & $\begin{array}{c}\text { Consulta } \\
\text { último mes }\end{array}$ & $\%$ & $\begin{array}{l}\text { Significancia } \\
\text { Ji-Cuadrado }\end{array}$ \\
\hline \multirow{4}{*}{$\begin{array}{l}\text { Grupos de } \\
\text { Edad }\end{array}$} & menores de 5 años & 424 & 90 & 21 & \multirow{4}{*}{0,686} \\
\hline & $5-19$ años & 1746 & 408 & 23 & \\
\hline & 20 - 64 años & 4383 & 1044 & 24 & \\
\hline & 65 y más años & 955 & 224 & 23 & \\
\hline \multirow[b]{2}{*}{ Sexo } & Femenino & 3598 & 937 & 26 & \multirow{2}{*}{0,428} \\
\hline & Masculino & 3925 & 831 & 21 & \\
\hline \multirow{2}{*}{ Estado civil } & Con pareja & 2880 & 685 & 24 & \multirow{2}{*}{0,333} \\
\hline & Otros & 4635 & 1081 & 23 & \\
\hline \multirow{2}{*}{$\begin{array}{l}\text { Zona de } \\
\text { residencia }\end{array}$} & Urbano & 4911 & 1172 & 24 & \multirow{2}{*}{0,308} \\
\hline & Rural & 2612 & 596 & 23 & \\
\hline \multirow{6}{*}{$\begin{array}{l}\text { Región de } \\
\text { residencia }\end{array}$} & Central & 4913 & 1198 & 24 & \multirow{6}{*}{0,000} \\
\hline & Chorotega & 546 & 173 & 32 & \\
\hline & Pacifico Central & 441 & 69 & 16 & \\
\hline & Brunca & 653 & 96 & 15 & \\
\hline & Huetar Atlántica & 800 & 203 & 25 & \\
\hline & Huetar Norte & 170 & 29 & 17 & \\
\hline \multirow{4}{*}{ Educación } & Hasta secundaria incompleta & 5192 & 1212 & 23 & \multirow{4}{*}{0,197} \\
\hline & $\begin{array}{l}\text { técnica completa } \\
\text { Parauniversitaria y }\end{array}$ & 1002 & 220 & 22 & \\
\hline & universitaria incompleta & 828 & 210 & 25 & \\
\hline & Universidad completa & 428 & 112 & 26 & \\
\hline \multirow{4}{*}{ Ocupación } & Patrono o socio activo & 137 & 34 & 25 & \multirow{4}{*}{0,826} \\
\hline & ${ }^{\star}$ Cuenta propia & 542 & 133 & 25 & \\
\hline & Empleado del estado & 702 & 182 & 26 & \\
\hline & ${ }^{* *}$ Empleado privado & 1398 & 346 & 25 & \\
\hline \multirow{5}{*}{$\begin{array}{l}\text { Quintiles } \\
\text { ingreso } \\
\text { percapita*** }\end{array}$} & I (US 5,28-74,05) & 1410 & 327 & 23 & \multirow{5}{*}{0,037} \\
\hline & II (US 74,36-118,03) & 1371 & 303 & 22 & \\
\hline & III (US 118,59-185,49) & 1295 & 291 & 22 & \\
\hline & IV (US $186,26-278,80)$ & 1148 & 308 & 27 & \\
\hline & $V($ US $291,04-1668,61)$ & 1112 & 248 & 22 & \\
\hline
\end{tabular}


Consulta ambulatoria o consulta médica

De las personas encuestadas que utilizaron los servicios de atención ambulatoria, un 23,5\% declaró haber asistido al menos a una consulta médica en el último mes. Con respecto a la última consulta, el $81 \%$ acceso a la seguridad social, $12,4 \%$ lo hicieron en un centro privado y el resto bajo otras modalidades. Los motivos de esta última consulta fueron, búsqueda de un diagnóstico o tratamiento a su dolencia (56 \%), revisión (39 \%) y el resto para medicación y otros motivos.

Entre los factores que predisponen la utilización de los servicios de consulta médica, están, el sexo (53\% de los que consultaron eran mujeres), el nivel educativo (parauniversitario y/o universitario) y el ingreso per cápita (IV quintil: US 186,26-278,80 mensuales), mientras que no se observó diferencias en los grupos de mayor edad (Tabla 1).

La prueba de Kruskal-Wallis, evidenció una asociación significativa entre las variables aseguramiento, zona y región de residencia y el tiempo en llegar a la última consulta, por lo que estas variables se pueden considerar factores que determinan el uso de la consulta ambulatoria en la población costarricense (Tabla 2).

Tabla 2. Uso del servicio de consulta ambulatoria y significancia observada, según variables facilitadoras

\begin{tabular}{|c|c|c|c|c|c|}
\hline \multirow{2}{*}{ Variable } & \multirow{2}{*}{ Categoría } & \multicolumn{4}{|c|}{$\begin{array}{l}\text { Tiempo en llegar a la consulta última consulta } \\
\text { (minutos) }\end{array}$} \\
\hline & & Número & Promedio & $\begin{array}{l}\text { Desviación } \\
\text { Estándar }\end{array}$ & $\begin{array}{l}\text { Significancia } \\
\text { Kruskal-Wallis }\end{array}$ \\
\hline \multirow{2}{*}{ Aseguramiento } & Sin seguro & 231 & 24 & 24 & \multirow[b]{2}{*}{0,000} \\
\hline & Con seguro & 1528 & 31 & 35 & \\
\hline \multirow{2}{*}{$\begin{array}{l}\text { Zona de } \\
\text { residencia }\end{array}$} & Urbano & 1172 & 32 & 36 & \multirow[b]{2}{*}{0,000} \\
\hline & Rural & 596 & 27 & 30 & \\
\hline \multirow{6}{*}{$\begin{array}{l}\text { Región de } \\
\text { residencia }\end{array}$} & Central & 1198 & 30 & 32 & \multirow{6}{*}{0,000} \\
\hline & Chorotega & 173 & 25 & 24 & \\
\hline & Pacifico Central & 69 & 24 & 19 & \\
\hline & Brunca & 96 & 37 & 48 & \\
\hline & Huetar Atlántica & 203 & 33 & 43 & \\
\hline & Huetar Norte & 29 & 37 & 50 & \\
\hline \multirow{5}{*}{$\begin{array}{l}\text { Quintiles ingreso } \\
\text { percapita }^{\star}\end{array}$} & I (US 5,28-74,05) & 327 & 32 & 39 & \multirow{5}{*}{0,489} \\
\hline & II (US 74,36-118,03) & 303 & 29 & 33 & \\
\hline & III (US 118,59-185,49) & 291 & 29 & 28 & \\
\hline & IV (US $186,26-278,80)$ & 308 & 30 & 32 & \\
\hline & $V$ (US 291,04-1668,61) & 248 & 30 & 36 & \\
\hline
\end{tabular}

*K-W Kruskal - Wallis 
Con la prueba de Ji-Cuadrado, se encontró que las variables región de residencia y quintil de ingreso per cápita, se asocian significativamente $(\mathrm{p}<0.05)$ con lo que denominamos factores que predisponen a la utilización de la consulta ambulatoria.

Cuando hablamos de factores facilitadores, las variables región de residencia y condición de aseguramiento, son las que presentan mayores diferencias con respecto al tiempo necesario para llegar al lugar donde se recibió la última consulta.

La prueba de Ji-Cuadrado, evidenció que existe asociación positiva entre la consulta ambulatoria y las variables de necesidad de utilizar los servicios $(\mathrm{p}=0,00)$, aproximadas por la salud autopercibida, el haber permanecido en cama más de medio día y el padecer una enfermedad crónica, consideradas estas como factores de necesidad.

\section{Hospitalización}

Un 5,3\% de los encuestados declaró haber estado hospitalizado al menos un día en el último año. El $46 \%$ utilizaron los servicios de hospitalización para realizase algún procedimiento quirúrgico, el $27 \%$ para estudios diagnósticos o revisión de rutina y el $23 \%$ por algún tratamiento que no requirió intervención quirúrgica. Un $47 \%$ declaró haber estado hospitalizado al menos cuatro días o menos y un $24 \%$ más de 10 días. Un $86 \%$ de estos internamientos se efectuaron en un hospital de CCSS, un $12 \%$ en hospitales privados y el restante en el Instituto Nacional de Seguros. Los porcentajes más elevados de hospitalizaciones fueron obtenidos en la región de Chorotega (7 \%) y los más bajos en las regiones Pacífico Central y Huetar Norte $(4,1 \%$ en ambas).

Con la prueba de Ji-Cuadrado, se encontró que solo la variable educación, con un nivel de significación del $5 \%$, se puede considerar como factor predisponente del uso de los servicios de hospitalización (Tabla 3).

El tiempo promedio de espera para ser hospitalizado es menor en los usuarios sin seguro. En dos de las siete regiones que componen el país (Huetar Atlántica y Chorotega) las personas esperan menos para ser hospitalizadas.

El aseguramiento y la región de residencia se identificaron como factores facilitadores del uso del servicio de hospitalización según la prueba de KruskalWallis (Tabla 4). 
Respecto a las variables que miden la necesidad la prueba de Ji-Cuadrado revela que hay asociación entre el padecer una enfermedad crónica y el haber sido hospitalizado ( $\mathrm{p}=0,009)$, no así en los casos de estado de salud autopercibida $(p=0,361)$ y el haber estado en cama $(p=0,162)$.

Tabla 3. Uso del servicio de hospitalización y significancia observada, según variables de predisposición

\begin{tabular}{|c|c|c|c|c|c|}
\hline Variable & Categoría & Número & $\begin{array}{c}\text { Hospitalizado } \\
\text { últimos } 12 \\
\text { meses }\end{array}$ & $\%$ & $\begin{array}{l}\text { Significancia } \\
\text { Ji-Cuadrado }\end{array}$ \\
\hline \multirow{4}{*}{ Grupos de Edad } & menores de 5 años & 424 & 20 & 5 & \multirow{4}{*}{0,413} \\
\hline & 5 - 19 años & 1746 & 104 & 6 & \\
\hline & $20-64$ años & 4383 & 220 & 5 & \\
\hline & 65 y más años & 955 & 55 & 6 & \\
\hline \multirow{2}{*}{ Sexo } & Femenino & 3598 & 221 & 6 & \multirow{2}{*}{0,186} \\
\hline & Masculino & 3925 & 178 & 5 & \\
\hline \multirow{2}{*}{ Estado civil } & Con pareja & 2880 & 142 & 5 & \multirow{2}{*}{0,586} \\
\hline & Otros & 4635 & 256 & 6 & \\
\hline \multirow{2}{*}{$\begin{array}{l}\text { Zona de } \\
\text { residencia }\end{array}$} & Urbano & 4911 & 266 & 5 & \multirow{2}{*}{0,586} \\
\hline & Rural & 2612 & 133 & 5 & \\
\hline \multirow{6}{*}{$\begin{array}{l}\text { Región de } \\
\text { residencia }\end{array}$} & Central & 4913 & 256 & 5 & \multirow{6}{*}{0,226} \\
\hline & Chorotega & 546 & 38 & 7 & \\
\hline & Pacifico Central & 441 & 18 & 4 & \\
\hline & Brunca & 653 & 30 & 5 & \\
\hline & Huetar Atlántica & 800 & 50 & 6 & \\
\hline & Huetar Norte & 170 & 7 & 4 & \\
\hline \multirow{4}{*}{ Educación } & Hasta secundaria incompleta & 5192 & 287 & 6 & \multirow{4}{*}{0,043} \\
\hline & $\begin{array}{l}\text { Secundaria y secundaria } \\
\text { técnica completa }\end{array}$ & 1002 & 41 & 4 & \\
\hline & $\begin{array}{l}\text { Para universitaria y } \\
\text { universitaria incompleta }\end{array}$ & 828 & 53 & 6 & \\
\hline & Universidad completa & 428 & 15 & 4 & \\
\hline \multirow{7}{*}{ Ocupación } & Patrono o socio activo & 137 & 5 & 4 & \multirow{7}{*}{0,79} \\
\hline & Cuenta propia & 535 & 26 & 5 & \\
\hline & Empleado del estado & 702 & 36 & 5 & \\
\hline & Empleado privado & 1311 & 63 & 5 & \\
\hline & $\begin{array}{l}\text { Serviçio domestiço/no } \\
\text { remunerado/ignorado }\end{array}$ & 94 & 4 & 5 & \\
\hline & Trabajador no remunerado & 5 & 1 & & \\
\hline & Ignorado & 2 & & & \\
\hline \multirow{5}{*}{$\begin{array}{l}\text { Quintiles ingreso } \\
\text { percapita* }\end{array}$} & I (US 5,28-74,05) & 1410 & 84 & 6 & \multirow{5}{*}{0,264} \\
\hline & II (US 74,36-118,03) & 1371 & 69 & 5 & \\
\hline & III (US 118,59-185,49) & 1295 & 67 & 5 & \\
\hline & IV (US $186,26-278,80)$ & 1148 & 75 & 7 & \\
\hline & $V$ (US 291,04-1668,61) & 1112 & 52 & 5 & \\
\hline
\end{tabular}

*Tipo de cambio promedio, venta julio 2006 (515,4 colones)

Un $11.5 \%$ declaró haber utilizado los servicios de urgencias en los últimos doce meses. El $75 \%$ mencionaron que los familiares lo llevaron, el $17 \%$ que el médico de la consulta ambulatoria lo refirió al servicio de urgencias y el restante se sentía mal. Ninguno de los encuestados refirió haber utilizado los servicios de urgencias 
mas de tres veces, $68 \%$ asistió una vez, $13 \%$ dos veces y el restante $19 \%$ tres veces. Este tipo de servicios son utilizados más frecuentemente por los niños en edad escolar (5 a 9 años) y los adultos mayores (65 años y más). Desde un punto de vista territorial, las regiones con mayor utilización corresponden a Huetar Norte, Atlántica y Brunca.

Tabla 4. Uso del servicio de hospitalización y significancia observada, según variables facilitadoras

\begin{tabular}{|c|c|c|c|c|c|}
\hline \multirow[b]{2}{*}{ Variable } & \multirow[b]{2}{*}{ Categoría } & \multicolumn{4}{|c|}{$\begin{array}{c}\text { Tiempo en lista de espera para hospitalizar se } \\
\text { (días) }\end{array}$} \\
\hline & & Número & Promedio & $\begin{array}{l}\text { Desviación } \\
\text { estándar }\end{array}$ & $\begin{array}{c}\text { Significancia } \\
\text { Kruskal- } \\
\text { Wallis }\end{array}$ \\
\hline \multirow{2}{*}{ Aseguramiento } & Sin seguro & 26 & 109 & 139 & \multirow{2}{*}{0,0231} \\
\hline & Con seguro & 167 & 95 & 86 & \\
\hline \multirow{2}{*}{$\begin{array}{l}\text { Zona de } \\
\text { residencia }\end{array}$} & Urbano & 126 & 101 & 102 & \multirow{2}{*}{0,295} \\
\hline & Rural & 68 & 92 & 89 & \\
\hline \multirow{6}{*}{$\begin{array}{l}\text { Región de } \\
\text { residencia }\end{array}$} & Central & 123 & 105 & 95 & \multirow{6}{*}{0,013} \\
\hline & Chorotega & 15 & 91 & 155 & \\
\hline & Pacifico Central & 13 & 109 & 38 & \\
\hline & Brunca & 7 & 101 & 122 & \\
\hline & Huetar Atlántica & 33 & 65 & 2 & \\
\hline & Huetar Norte & 3 & 124 & 202 & \\
\hline \multirow{5}{*}{$\begin{array}{l}\text { Quintiles ingreso } \\
\text { percapita* }^{*}\end{array}$} & I (US 5,28-74,05) & 44 & 81 & 85 & \multirow{5}{*}{0,788} \\
\hline & II (US 74,36-118,03) & 41 & 90 & 154 & \\
\hline & III (US 118,59-185,49) & 21 & 95 & 109 & \\
\hline & IV (US 186,26-278,80) & 35 & 82 & 22 & \\
\hline & V (US 291,04-1668,61) & 29 & 83 & 94 & \\
\hline
\end{tabular}

Urgencias

La prueba de Ji-Cuadrado evidencia que la edad, el estado civil, la región de residencia, la educación y el quintil de ingreso per cápita predisponen el uso de servicios de urgencias.

Con la prueba de Ji-Cuadrado, se encontró asociación entre el uso del servicio de urgencias y el haber estado en cama a lo sumo medio día ( $\mathrm{p}=0,04)$, y que para la población costarricense las variables de enfermedad crónica y una percepción mala o regular de su salud, no se asocian a factores de necesidad que condicionan la utilización de dichos servicios ( $p>0,05)$.

No se pudieron determinar variables con características de factores facilitadores en los servicios de urgencias, dado que la encuesta no presenta información sobre el tiempo para recibir este servicio. 
Tabla 5. Uso del servicio de urgencias y significancia observada, según variables de predisposición

\begin{tabular}{|c|c|c|c|c|c|}
\hline Variable & Categoría & Número & $\begin{array}{c}\text { Asistió a } \\
\text { urgencias último } \\
12 \text { meses }\end{array}$ & Porcentaje & $\begin{array}{l}\text { Significancia } \\
\text { Ji-Cuadrado }\end{array}$ \\
\hline \multirow{4}{*}{$\begin{array}{l}\text { Grupos de } \\
\text { Edad }\end{array}$} & menores de 5 años & 424 & 45 & 11 & \multirow{4}{*}{0,001} \\
\hline & 5 - 19 años & 1746 & 238 & 14 & \\
\hline & 20 - 64 años & 4383 & 454 & 10 & \\
\hline & 65 y más años & 955 & 125 & 13 & \\
\hline \multirow{2}{*}{ Sexo } & Femenino & 3598 & 455 & 13 & \multirow{2}{*}{0,731} \\
\hline & Masculino & 3925 & 408 & 10 & \\
\hline \multirow{2}{*}{ Estado civil } & Con pareja & 2880 & 296 & 10 & \multirow{2}{*}{0,006} \\
\hline & Otros & 4635 & 566 & 12 & \\
\hline \multirow{2}{*}{$\begin{array}{l}\text { Zona de } \\
\text { residencia }\end{array}$} & Urbano & 4911 & 561 & 11 & \multirow{2}{*}{0,857} \\
\hline & Rural & 2612 & 302 & 12 & \\
\hline \multirow{6}{*}{$\begin{array}{l}\text { Región de } \\
\text { residencia }\end{array}$} & Central & 4913 & 555 & 11 & \multirow{6}{*}{0,003} \\
\hline & Chorotega & 546 & 54 & 10 & \\
\hline & Pacifico Central & 441 & 31 & 7 & \\
\hline & Brunca & 653 & 91 & 14 & \\
\hline & Huetar Atlántica & 800 & 108 & 14 & \\
\hline & Huetar Norte & 170 & 24 & 14 & \\
\hline \multirow{4}{*}{ Educación } & $\begin{array}{l}\text { Secundaria incompleta } \\
\text { Secundaria y secundaria }\end{array}$ & 5192 & 630 & 12 & \multirow{4}{*}{0,008} \\
\hline & $\begin{array}{l}\text { técnica completa } \\
\text { Para universitaria y }\end{array}$ & 1002 & 98 & 10 & \\
\hline & universitaria incompleta & 828 & 93 & 11 & \\
\hline & Universidad completa & 428 & 32 & 7 & \\
\hline \multirow{5}{*}{ Ocupación } & Patrono o socio activo & 137 & 16 & 12 & \multirow{5}{*}{0,747} \\
\hline & Cuenta propia & 542 & 60 & 11 & \\
\hline & Empleado del estado & 702 & 65 & 9 & \\
\hline & Empleado privado & 1398 & 150 & 11 & \\
\hline & Servicio domestico & 87 & 12 & 14 & \\
\hline \multirow{5}{*}{$\begin{array}{l}\text { Quintiles } \\
\text { ingreso } \\
\text { percapita* }\end{array}$} & I (US 5,28-74,05) & 1410 & 189 & 13 & \multirow{5}{*}{0,049} \\
\hline & II (US 74,36-118,03) & 1371 & 155 & 11 & \\
\hline & III (US 118,59-185,49) & 1295 & 134 & 10 & \\
\hline & IV (US 186,26-278,80) & 1148 & 147 & 13 & \\
\hline & V (US 291,04-1668,61) & 1112 & 116 & 10 & \\
\hline
\end{tabular}

${ }^{*}$ Incluye al grupo de no remunerado e ignorado; ${ }^{* *}$ Incluye servicios domésticos

\section{DISCUSIÓN}

En términos globales las variables que postula el modelo de Andersen y Newman sobre la utilización de servicios de salud, se cumplen para Costa Rica. No obstante la significancia de los factores varía entre los servicios de consulta ambulatoria, hospitalización y urgencias.

Las tasas de utilización de servicios de salud son difíciles de comparar entre Costa Rica y otros países, ya que el tiempo evaluado en las preguntas varía de 15 días a 1 año. Pero el análisis comparativo si permite evidenciar que al aumentar el periodo de estudio se aumentan las tasas de utilización (23-25).

En la utilización de la consulta ambulatoria y urgencia, coinciden como factores predisponentes la región de residencia y el quintil de ingreso percápita. 
Con respecto a la región de residencia es importante identificar las causas de las diferencias geográficas en utilización, dado que se puede tratar de aspectos culturales, otras veces responden a la disponibilidad de servicios y las condiciones de acceso definidas por el grado de ruralidad predominante en cada zona.

Solo la educación es coincidente como factor determinante en el uso del servicio de hospitalización y de urgencias. Este resultado no concuerda con resultados de otros países con sistema de salud de cobertura universal o casi universal, dado que estos servicios estarían determinados por la gravedad y urgencia y no por el nivel de estudio (26).

Resultó coincidente que las variables de aseguramiento y región de residencia se asocian a factores facilitadores de la utilización de los servicios de consulta ambulatoria y hospitalización.

Estudios realizados en otros países han mostrado que el principal número de visitas para el cuidado de la salud son al médico general y que las personas pertenecientes a niveles socioeconómicos más bajos presentan una mayor utilización de tales servicios (27-29).

Todas las variables de necesidad estudiadas se asociaron con la utilización de los servicios de consulta ambulatoria, pero solo la enfermedad crónica se asoció con la hospitalización y el haber permanecido en cama con el uso de los servicios de urgencia.

Por lo anterior, las estrategias para enfrentar los diferentes factores que condicionan la utilización de servicios de salud deben enfocarse en función del servicio, pues se evidenció que no son los mismos factores para consulta ambulatoria, hospitalización o urgencia.

Para completar el análisis de los determinantes de la utilización de servicios de salud se recomienda realizar un análisis multivariado para controlar por las variables asociadas. En la literatura se han estudiado básicamente tres métodos estadísticos alternativos para analizar las diferencias en utilización de servicios de salud $(30,31)$. El primer caso se refiere a un modelo donde la variable dependiente sería el número de consultas para lo que se supone que la utilización de servicios de salud es una decisión determinada por el paciente (regresiones tipo binomial negativa). La segunda alternativa se basa en la teoría económica del agente-principal (modelo logit y binomial negativa). La tercera alternativa se refiere a los modelos multinivel que permiten el control de la heterogeneidad individual. 
También se recomienda para futuros estudios realizar un análisis separado para niños y adultos, dadas las diferencias en el perfil epidemiológico de estas dos poblaciones. Yestudiar por separado las consultas ambulatorias de revisión donde el individuo no está enfermo, de aquellas por problemas de salud (dolencias).

Las principales limitaciones están asociadas al tipo de estudio utilizado (observacional), el sesgo de memoria inherente a las encuestas y la posible subjetividad del autoreporte de las necesidades de salud .

Agradecimientos. Este proyecto contó con el apoyo de una beca parcial sin condiciones del programa académico del PPPI de la Merck Company Foundation, brazo filantrópico de Merck \& Co. Inc., Whitehouse Station, New Jersey, EE UU.

\section{REFERENCIAS}

1.Contextualización de la utilización de servicios de salud [Internet]. Caja Costarricense del Seguro Social. Disponible en: http://www.ccss.sa.cr/html/organizacion/gestion/gerencias/ medica/ germed/dis/diess/indica02.htm. Consultado febrero del 2009.

2. Utilización de servicios de salud [Internet] Caja Costarricense del Seguro Social. Disponible en: http://www.ccss.sa.cr/html/organizacion/gestion/gerencias/administrativa/dcss/archivos/ estudios_realizados/UTILIZACIONDESERVICIOSDESALUD.pdf.Consultado febrero del 2009.

3. Rodríguez A, Castillo J. ¿Indicios de segmentación en el sistema de salud Costarricense?. Informe del Estado de la Nación en Desarrollo Sostenible. 2008; 14:102-103.

4. Andersen R, Newman JF. Societal and individual determinants of medical care utilization in the United States. Millbank Memorial Fund Q 1973; 51:95-124.

5. Andersen, R. Revisiting the Behavioral Model and Access to Medical Care: Does it Matter?. Journal of Health and Social Behavior 1995; 36 (1): 1-10.

6. Dever A. Epidemiología y administración de servicios de salud. En: Epidemiología de la utilización de los servicios de salud. Organización Panamericana de la Salud; 1991.

7. Borrell C, Fernández E. Desigualdades de utilización. Estado de la cuestión y estrategias de futuro. En: López Casasnovas G, Callau J (eds.). XIX Jornadas de Economía de la Salud: necesidad sanitaria, demanda y utilización; Zaragoza: Asociación de economía de la salud; 1999. Pp. $171-84$.

8. Sánchez J, Cavanillas A. Factores asociados al uso inadecuado de urgencias hospitalario. Emergencias 2005; 17:138-144.

9. Aday LA, Andersen R. A framework for the study of access to medical care. Health Serv Res 1974; 9(3):208-220.

10. Fiedler J. A review of the literature on access and utilization of medical care with special emphasis on rural primary care. Soc Sci Med 1981; 15:129-142.

11. Newhouse J, Phelps CE. Policy options and the impact of national health insurance. J Calif Dent Asocc 1974; 2(10):53-64.

12. Grossman M. On the content of health capital and the demand for health. J Polit Econ 1972; 80:223-255.

13. Hulka B, Wheat J. Patterns of utilization. Med Care 1985; 23(5):438-460.

14. Davis K, Gold M, Makuc D. Access to health care for the poor: Does the gap remain. Annu Rev Public Health 1981; 2:159-82. 
15. Clancy CM, Eisenberg JM. Emergency Medicine in Population-Based Systems of Care. Ann Emerg Med 1997; 30:800-3.

16. Aday LA, Andersen R. Insurance coverage and access: Implications for health policy Health Serv Res 1978; 13(4):369-377.

17. Leibowitz A, Friedman B. Familiy requests and the derived demand for health care. Econ Inq $1979 ; 17: 133-139$.

18. Andersen R. Health status indices and access to medical care. Am J Public Health 1978; 68(5):458-463.

19. Alvarez, Begoña. La demanda atendida de consultas médicas y servicios urgentes en España. Investigaciones Económicas 2001; 25(1): 93-138.

20. Fabbri D, Monfardini C. Public vs. Private Health Care Services Demand in Italy. Giornale degli Economisti. 2003; 62(1): 93-123.

21. Valencia-Mendoza A, Bertozzi S. A predictive model for the utilization of curative ambulatory health services in Mexico. Salud Pública de México 2008; 50(5): 397-407.

22. Encuesta Nacional de Salud (ENSA2006) [Internet]. Centro Centroamericano de Población. Disponible en: http://www.ccp.ucr.ac.cr/farmacoeconomia/docEnsa.html. Consultado Julio del 2007.

23. Palo Stoller E. Patterns of physician utilization by the elderly: A multivariate analysis. Med Care 1992;20:1080-1089.

24. Panamerican Health Organization. Health conditions in the Americas 1994. PAHO Washington, D.C. 1994; 549: 55-56.

25. Borges-Yañez SA, Gómez-Dantés H. Uso de los servicios de salud por la población de 60 años y más en México. Salud pública de méxico. 1998, 40(1): 1-11.

26. Lostao L, Regidor E, Calle ME, Navarro P, Domínguez V. Evolución de las diferencias socioeconómcias en la utilización y accesibilidad de los senvicios sanitarios en España entre 1987 Y 1995/97. Rev Esp Salud Pública. 2001; 75(2):115-128.

27. Piperno A, Di Orio F. Social differences in health and utilization of health services in Italy. Soc Sci Med 1990; 31:305-312.

28. Haynes R. Inequalities in health and health service use: evidence from the General Household Survey. Soc Sci Med 1991; 33:361-368.

29. Van der Meer JBW, Van den Bos J, Mackenbach JP. Socioeconomic differences in the utilization of health services in a Dutch population: the contribution of health status. Health Policy 1996; 37:1-18.

30. Jiménez S, Labeaga JM, Martínez M. Latent class versus two part models in the demand for physician services across the European Union. Health Economics. 2002; 11(4): 301-321.

31. Deb P, Trivedi P. The structure of demand for health care: latent class versus two-part models Journal of Health Economics. 2002; 21(4): 601-625. 